\title{
Preparation of cellulose nanocrystals from lignin-rich reject material for oil emulsification
}

in an aqueous environment

\section{Jonna Ojala ${ }^{a^{*}}$, Juho A. Sirviö ${ }^{a}$, and Henrikki Liimatainen ${ }^{a}$}

\author{
*Jonna Ojala, Researcher, jonna.ojala@oulu.fi (Corresponding author) \\ Juho Sirviö, Senior Researcher, juho.sirvio@oulu.fi \\ Henrikki Liimatainen, Associate Professor, henrikki.liimatainen@oulu.fi \\ aUniversity of Oulu, Fibre and Particle Engineering, P.O. Box 4300, FI-90014 Oulu, Finland
}

\section{Abstract}

Cellulose nanocrystals (CNCs) with amphiphilic features were used in oil drop stabilization in diesel oil-in-water (o/w) emulsion. The functionalized CNCs were synthesized from a lignin-rich reject cellulose source from the pulp and paper industry, i.e., the non-bleached fines fractions of carton pulp. Partial periodate-chlorite oxidation, which was followed by reductive butylamination, was used to obtain surface-modified amphiphilic CNCs. All studied CNCs prevented droplet coalescence by stabilizing oil droplets in the emulsion thus resulting in stable o/w Pickering-like emulsions. CNCs from the fines fractions at concentrations $0.05-0.1 \%$ (weight by weight, $w / w$ ) provided high stability against creaming (i.e., phase separation), and they did not de-emulsify at low temperatures since the oil droplet size remained small at $+5{ }^{\circ} \mathrm{C}$ at a $0.05 \%(\mathrm{w} / \mathrm{w}) \mathrm{CNC}$ concentration. Salinity improved the stability against creaming with the reference chemical pulp CNC, but negatively affected the emulsion creaming rate for CNCs that had a higher level of lignin. However, the nonbleached fines fraction of the pulp may provide one potential and cost-effective raw material source for the development of a novel bio-based chemical.

\section{Keywords}

Nanocellulose, Reject fiber fines, Cellulose nanocrystals, Oil in water emulsion, Bio-based dispersant, Lignin 
32

33

34 Ever increasing oil activities, including oil drilling and oil transportation by sea, increase the

\section{Introduction} risk of oil spills that may be detrimental to the marine ecosystem (Al-Majed et al. 2012). In

the larger, nearer to the coast spills, the risk of oil floating into shallow waters is greater, and more severe damage to the environment is likely to occur. The use of surface active oil dispersants is often the most often deployed response option for large spills. Such dispersants enhance the naturally occurring oil slick breaking action caused by wind and waves to obtain sufficiently small droplets to aid microbial degradation (Zeinstra-Helfrich et al. 2015). An effective oil dispersant breaks the oil into small droplets, typically less than 70 microns in diameter, while simultaneously lowering the oil droplet rise velocity and enabling the potential microbial attack before the droplet reaches the ocean's surface (Yu et al. 2014; Prince 2015). Dispersion of oil has reportedly promoted the biodegradation of the oil in the case of deep water spills and also reduced the impact of floating oil on the shoreline (Prince 2015).

Most commercially available chemical dispersants are a mixture of both non-ionic and anionic surfactants, as well as other solvents, which makes the dispersant partially soluble in both oil and water (Al-Majed et al. 2012; Guodong et al. 2015; Nyankson et al. 2015). Synthetic dispersants have been used for decades, even though they appear to have a moderate level of toxicity that adversely affects marine ecosystems (George-Ares and Clark 2000; Zhang et al. 2013; Prince 2015). Therefore, research on new, sustainable, and more 
53 benign dispersants based on natural raw materials is highly desirable. In particular, different

54 bio-based reject materials originating from industrial processes, such as papermaking and

55 biomass processing, are attractive due to their abundance, low cost, and natural

56 biodegradability(Silva et al. 2008; García et al. 2016; Orelma et al. 2017). For example, fiber

57 fines (the small particle size fractions of cellulose pulps), which are typically related to the

58 increased smoothness of paper and board, as well as improved air permeability resistance,

59 are quite problematic in some papermaking processes, such as producing packaging board.

60 The effect of fines on paper and board processing stages, like dewatering and retention, is

61 negative, and when the content of fines increases, the strength properties are getting worse

62 due to poor binding capability of fines. In addition, fiber fines often contain pitch-like

63 materials (stickies), which cause holes and other problems in the manufacture of paper

64 products. Paper and carton manufacturers, especially those working with mechanical

65 pulping, where lignin-rich fines are stiff and do not bond to fibers, find these fines fractions

66 to be less desirable fractions (Gharehkhani et al. 2015). These side-stream fractions are

67 often considered as reject material; therefore, novel applications for the utilization of non-

68 bleached fiber fines are desirable.

69 Nanomaterials are typically considered to be materials which are in the form of particles,

70 tubes, fibers or rods and have at least one dimension between 1 to $100 \mathrm{~nm}$. Technologies

71 that include different nanosized materials, such as nanowires, magnetic composites/gels,

72 or bio-based nanoparticles, may provide alternative methods to control oil spills in the

73 marine environment(Saha et al. 2013; Wang et al. 2013; Pi et al. 2016; Daza et al. 2017)

74 These applications are based on tuned hydrophobicity through creating complexes or 
75 surface coatings that enable oil adsorption. Bio-based nanoparticles, such as cellulose

77 dispersants for enhanced oil spill recovery. However, naturally hydrophilic cellulose typically requires chemical modifications, including the attachment of hydrophobic functional groups, to improve its ability to stabilize o/w emulsions (Xhanari et al. 2011). Naturally, more hydrophobic raw materials such as lignin-rich fiber fines provide a raw material that is cheaper and is assumed to function better on hydrophobic matrices.

The ability of different kinds of nanoparticles to adsorb at interfaces is a known phenomenon that has been utilized broadly in the emulsion stabilization in so-called Pickering emulsions (Kalashnikova et al. 2011, 2013; Chevalier and Bolzinger 2013; Jiang et al. 2014; Hu et al. 2015a, 2015b). Within nanoparticle stabilized emulsions, the creaming phenomenon has been reported as the most probable route for de-stabilization. Creaming refers to the separation of phases while the oil droplet size remains stable in the floating creamy oil phase without oil droplet coalescence. Amphiphilic bifunctionalized cellulose nanoparticles are able to form Pickering-like emulsions, stabilizing the oil droplet against coalescence by adsorbing into the oil water interface covering the oil droplet (Ojala et al. 2016). Moreover, those functionalized nanoparticles are predicted to slow down the creaming phenomenon, thus, potentially make the natural biodegradation of oil more efficient.

In this work, amphiphilic cellulose nanocrystals (CNC) were used as dispersing and stabilizing agents in diesel $\mathrm{o} / \mathrm{w}$ emulsions in an aqueous environment. The cellulose nanocrystals were synthesized from lignin-rich fiber fines, a side product of carton 
97 production, through partial periodate-chlorite oxidation and reductive amination, followed by a homogenization phase to liberate individual bifunctionalized cellulose nanocrystals

99 (Sirvio et al. 2011). Unbleached fines fractions have high lignin content believed to promote 100 their inherent hydrophobicity and feasibility for oil dispersion and stabilization material 101 fabrication. Altogether, three different samples were fabricated. Two cellulose nanocrystal 102 samples were made from the lignin-rich, semi-chemical pulp fines fractions CNC-Lig-1 and 103 CNC-Lig-2. One sample was made from a low-lignin birch pulp (Kraft pulp) (CNC-CHEM). The 104 CNC suspensions were characterized with a transmission electron microscope (TEM), while 105 the dispersion effectiveness was addressed by measuring the oil droplet size in emulsion 106 along with the emulsion stability. The oil droplet size and o/w emulsion stability 107 measurements were conducted at a room temperature of $20^{\circ} \mathrm{C}$ and at $5^{\circ} \mathrm{C}$ to simulate the 108 colder water environment.

\section{Materials and Methods}

\subsection{Materials}

114 Semi-chemical hardwood pulp from corrugated board manufacturing was obtained from a

115 Finnish mill as a readily refined pulp suspension from which a fines fraction was separated 116 for nanocellulose synthesis. Kraft pulp, i.e., chemical birch pulp (Betula Pendula), was 117 received as dry sheets and was then disintegrated into water according to standard 118 procedure (ISO 5263-1:2004). The chemical composition of the cellulose materials that were 
119 used was determined according to standard procedures for wood extractives (SCAN-CM 120 49:03 standard), hemicelluloses (TAPPI-T Method 212 om-02), and lignin (TAPPI-T Method 121222 om-02).

122 All of the chemicals used in the periodate and chlorite oxidations $\left(\mathrm{NaIO}_{4}, \mathrm{NaClO}_{2}\right.$, and $\left.123 \mathrm{CH}_{3} \mathrm{COOH}\right)$ and aldehyde and carboxyl content analyses $\left(\mathrm{NH}_{2} \mathrm{OH} \cdot \mathrm{HCl}, \mathrm{CH}_{3} \mathrm{COONa} \cdot 2 \mathrm{H}_{2} \mathrm{O}\right.$, $124 \mathrm{NaCl}$, and $\mathrm{NaOH}$ ) were pro analysis (p.a.) grade obtained from Sigma-Aldrich (Germany). In 125 the reductive amination reaction, 2-picoline borane (Sigma-Aldrich, St. Louis, MO, USA 126 [95\%]) and $n$-butylamine hydrochloride (Tokyo Chemical Industry, Belgium [>98\%]) were 127 used. Ethanol ( $\mathrm{C}_{2} \mathrm{H}_{6} \mathrm{O}$; purity $\left.\sim 96 \%\right)$, obtained from VWR International GmbH (Finland), and 128 hydrochloric acid ( $\mathrm{HCl}$ ) from Sigma-Aldrich (Germany) were used in the washing steps after 129 the amination process. All chemicals were p.a. grade and were used without further 130 purification. Deionized water was used in all dilutions and washing steps throughout the 131 experiments. Lightweight, sulphur-free, winter grade marine diesel oil (Neste Oil, Finland) 132 with a density of $828 \mathrm{~kg} / \mathrm{m}^{3}$ at $15^{\circ} \mathrm{C}$ and a viscosity of $1.846 \mathrm{~mm}^{2} \mathrm{~s}^{-1}$ at $40{ }^{\circ} \mathrm{C}$ was used for 133 all o/w emulsions.

\subsection{Preparation of synthetic seawater}

136 To mimic oceanic conditions, synthetic seawater was prepared according to the modified

137 formula of Kester et al. (1967). The composition of synthetic seawater is presented in Table

138 1. Briefly, the gravimetric salts were used in anhydrous form after drying them overnight in 139 an oven. Reagents containing the water of hydration were first weighed, then diluted to be 
140 used as a stock solution. The $\mathrm{pH}$ of the readily made seawater was 8.5 , conductivity was $14119.3[\mathrm{mS} / \mathrm{cm}]$, and salinity was 3.5\%. The freshly prepared seawater was sterilized $121^{\circ} \mathrm{C}$ at

1421 bar for 15 min to prevent any microbial growth.

\begin{tabular}{ccc}
\hline Gravimetric salts & Amount [g/kg of the solution] & Concentration [mmol/L] \\
\hline $\mathrm{NaCl}$ & 23.926 & 409.41 \\
$\mathrm{Na}_{2} \mathrm{SO}_{4}$ & 4.008 & 28.22 \\
$\mathrm{KCl}$ & 0.677 & 9.08 \\
$\mathrm{NaHCO}$ & 0.196 & 2.33 \\
$\mathrm{KBr}$ & 0.098 & 0.82 \\
$\mathrm{H}_{3} \mathrm{BO}_{3}$ & 0.026 & 0.42 \\
$\mathrm{NaF}$ & 0.003 & 0.07 \\
\hline Volumetric salts & Concentration [mmol/ L] & \\
\hline $\mathrm{MgCl}_{2} \cdot 6 \mathrm{H}_{2} \mathrm{O}$ & 53.7 & \\
$\mathrm{CaCl}_{2} \cdot 2 \mathrm{H}_{2} \mathrm{O}$ & 10.33 & \\
$\mathrm{SrCl}_{2} \cdot 6 \mathrm{H}_{2} \mathrm{O}$ & 0.09 & \\
\hline
\end{tabular}

143

144 Table 1. Composition of synthetic seawater.

145

146 2.3. Fabrication of butylamino-functionalized CNCS

147 2.3.1 Separation of a fines fraction from semi-chemical pulp

148 A fraction containing only the fines material (particles $<150 \mu \mathrm{m}$ ) was separated from the 149 semi-chemical pulp by screening with a 100 mesh screen under constant water flow. The 150 suspension was then concentrated through a $20 \mu \mathrm{m}$ filtering cloth to obtain a sample with 151 a dry matter content of approximately $10 \%$. This material was used to fabricate 152 functionalized CNCs. 


\subsubsection{The chemical oxidation-amination pre-treatment}

155 The bifunctionalized CNCs with amphiphilic nature were prepared using a combination of 156 oxidative chemical pre-treatments, followed by amination with butylamine and finally a 157 mechanical homogenization phase, to liberate the individual nanocrystals.

158 The fabrication was initiated by oxidizing the fines fraction of the semi-chemical pulp with 159 metaperiodate to result in two diverse aldehyde contents (DAC_A and DAC_B). In the 160 procedure, wet cellulose pulp (15 g oven-dry weight) was oxidized using $12.3 \mathrm{~g}$ of sodium 161 periodate $\left(\mathrm{NaIO}_{4}\right)$ in deionized water $(1,500 \mathrm{ml})$ at $65^{\circ} \mathrm{C}$ for $180 \mathrm{~min}$ to attain DAC_A. DAC_B 162 was prepared similarly, except that $27 \mathrm{~g}$ of lithium chloride ( $\mathrm{LiCl}$ ) was used as an additive 163 and the temperature setting was $75^{\circ} \mathrm{C}$. The third product, DAC_C, was prepared using 164 similar chemistry as that used for the DAC_B, but Kraft pulp was used as a raw material.

165 In the second step, sodium chlorite $\left(\mathrm{NaClO}_{2}\right)$ was used to partially oxidize the aldehydes of 166 the DACs to obtain dicarboxylic acid groups (PO-DACs). The amounts of sodium chlorite 167 used were $0.497 \mathrm{~g}, 0.80 \mathrm{~g}$, and $0.80 \mathrm{~g}$ for DAC_A, DAC_B, and DAC_C, respectively. Sodium 168 chlorite was first diluted with $17.78 \mathrm{~g}$ of deionized water, and then poured into a beaker 169 with $26.65 \mathrm{ml}$ of 20 vol\% acetic acid. These solutions were then mixed with $2 \mathrm{~g}$ of DAC, 170 oxidized, and then diluted in $44.4 \mathrm{ml}$ of water. The oxidation reaction was completed under 171 a fume hood with continuous mixing for $8 \mathrm{~min}$. The three cellulose products obtained were 172 labeled PO-DAC/A, PO-DAC/B, and PO-DAC/C.

173 Reductive amination was then used to introduce $n$-butylamino groups to the oxidized 174 cellulose. The amination for DAC_A was conducted by adding $7.37 \mathrm{~g} n$-butylamine 
175 hydrochloride to $200 \mathrm{ml}$ of deionized water and adjusting the $\mathrm{pH}$ to 4.5 with a diluted $\mathrm{HCl}$

176 solution. For DAC_B and DAC_C, $12.19 \mathrm{~g}$ of $n$-butylamine were used. Next, a 2-picoline 177 borane solution, where $1.44 \mathrm{~g}$ (for DAC_A) and $2.38 \mathrm{~g}$ (for both DAC_B and DAC_C) had been 178 diluted in $200 \mathrm{ml}$ deionized water, was added to the $n$-butylamine hydrochloride solution, 179 and $4 \mathrm{~g}$ of each PO-DAC fiber sample were also weighed. After $72 \mathrm{~h}$ of continuous mixing at 180 room temperature, the samples were filtered to stop the reaction and were washed with 181 water $(\mathrm{V}=200 \mathrm{ml})$ and ethanol $(\mathrm{V}=300 \mathrm{ml})$. Finally, the solid was collected into a beaker, 182 and a $200 \mathrm{ml} 0.1 \mathrm{M} \mathrm{HCl}$ solution was added. After 5 min of mixing, the suspension was 183 filtered and washed with $500 \mathrm{ml}$ of deionized water. This procedure resulted in three 184 different bifunctionalized samples.

\subsubsection{Liberation of nanoparticles from chemically pre-treated celluloses}

187 The bifunctionalized cellulose materials were converted to individual CNCs with a solids 188 content of $0.1 \mathrm{wt} \%$ and a $\mathrm{pH}$ of $10(\mathrm{pH}$ was adjusted using $\mathrm{NaOH})$ using a homogenization 189 treatment with an M-110EH-30 microfluidizer (Microfluidics, Newton, MA, USA). The 190 suspensions were passed once through a combination of a $400 \mu \mathrm{m}$ auxiliary processing 191 module (APM) and $200 \mu \mathrm{m}$ interaction chamber (IXC) at a pressure of 1,300 bar followed by 192 passage through a combination of $400 \mu \mathrm{m}$ APM and $100 \mu \mathrm{m} \mathrm{IXC} \mathrm{at} \mathrm{a} \mathrm{pressure} \mathrm{of} \mathrm{1,700} \mathrm{bar.}$

193 Finally, the cellulose nanoparticles were labelled as CNC-LIG-1, CNC-LIG-2, and CNC-CHEM 194 to indicate the bifunctionalized nanocrystals (CNC-LIG-1 and CNC-LIG-2 from the lignin-rich 195 semi-chemical pulp and CNC-CHEM from the Kraft pulp). 
197 2.4. Determination of initial aldehyde content and carboxyl content after oxidation pre-

198 treatment

199 The initial aldehyde content of celluloses after sodium periodate oxidation was analyzed

200 through an oxime reaction, which is described in more detail elsewhere (Sirvio et al. 2011).

201 Briefly, the reaction is based on the premise that one mole of aldehyde in oxidized cellulose

202 reacts with one mole of $\mathrm{NH}_{2} \mathrm{OH} \bullet \mathrm{HCl}$, and, after the reaction, the nitrogen content can be

203 determined from a freeze-dried sample by using an elemental analyzer (PerkinElmer

204 CHNS/O 2400 Series II; PerkinEImer, Waltham, MA, USA). The aldehyde content can then

205 be calculated straight from the known nitrogen content. The carboxyl content of the

206 dicarboxylic acid samples was determined with conductometric titration as described by

207 Katz et al. (1984) and Rattaz et al. (2011).

208

209 2.5. Determination of the amino groups of bifunctionalized celluloses

210 The samples were freeze-dried (Scanvac CoolSafe; Labogene Aps, Lygne, Denmark) at a

211 solids content of $0.1 \mathrm{wt} \%$ for $60 \mathrm{~h}$ prior to elemental analysis. The CHN/O 2400 organic

212 elemental analyzer (PerkinElmer, Waltham, MA, USA) was used to determine the nitrogen

213 content of the samples from which the number of amino groups was calculated. 
216 The morphological features of the fabricated nanocelluloses were analyzed with a Tecnai

217 G2 Spirit TEM (FEI Europe, Eindhoven, the Netherlands). Samples were prepared by diluting

218 each sample with ultrapure water. A small droplet of the dilution was added on top of a

219 Butvar-coated copper grid. The excess amount of the sample was removed from the grid by

220 touching the droplet with the corner of a filter paper. Negative staining of the samples was

221 performed by placing a droplet of uranyl acetate $(2 \% \mathrm{w} / \mathrm{v})$ on top of each specimen. The

222 excess uranyl acetate was removed with filter paper as described above. The grids were

223 dried at room temperature and analyzed at $100 \mathrm{kV}$ under standard conditions. Images were

224 captured using a Quemesa CCD camera and iTEM image analysis software (Olympus Soft

225 Imaging Solutions GMBH, Munster, Germany) to measure the width of the individual

226 nanocelluloses. In total, at least 50 crystals of each sample were measured. The final results

227 were averaged and standard deviations were calculated.

229 2.7. Formation of diesel o/w emulsion and droplet stabilization using cellulose nanocrystals

230 To determine the influence of the CNCs (CNC-LIG-1-2 and CNC-CHEM) on o/w 231 emulsification, the nanocelluloses were mixed with the water phase before oil was added 232 and dispersed. The final nanocellulose concentrations varied from $0.05 \%$ to $0.1 \%(\mathrm{w} / \mathrm{w})$. The 233 emulsions were prepared using the UltraTurrax mixer (IKA T25; IKA-Works, Staufen im 234 Breisgau, Germany) at 7,000 rpm for $15 \mathrm{~min}$ and maintaining the 1:10 oil to water ratio 235 throughout the study. To monitor the stability in cold conditions, some emulsions were 
236 prepared using cooled (approx. $5 \pm 2{ }^{\circ} \mathrm{C}$ ) water, and the samples were held in an ice bath to

237 prevent warming during dispersing.

239 2.8. Particle size analysis and microscopy

240 The changes in oil droplet size were analyzed using a laser diffraction particle size analyzer

241 (LS 13 320; Beckman Coulter, Indianapolis, IN, USA). Each sample was measured directly

242 after dispersing, and three parallel measurements were performed. Droplet sizes of 243 samples were observed and imaged using a LeicaMZ LIII stereomicroscope (Leica 244 Microsystems Ltd., Heerbrugg, Switzerland) to visualize oil droplet density, size, and 245 possible aggregation after dispersion.

247 2.9. Emulsion stability analysis with analytical centrifuge

248 An analytical centrifuge (LUMiFuge; LUM GmbH, Berlin, Germany) was used to evaluate the $249 \mathrm{O} / \mathrm{w}$ emulsion stability at room temperature and at a cold temperature $\left(5^{\circ} \mathrm{C}\right)$ mimicking the 250 conditions prevailing in cold seawaters. The centrifuge detects the emulsion stability and 251 demixing phenomena during centrifugation by measuring the changes in light 252 transmittance at an $800 \mathrm{~nm}$ wavelength over the whole sample height. A 1,200 rpm 253 rotational speed, corresponding to a relative centrifugal force of $205 \mathrm{G}$, was used. Changes 254 in transmission indicate phase separation through creaming. A measurement at $30 \mathrm{~min}$ 255 reveals the creaming process as a function of time, whereas lower transmission values 
256 indicate better stability against creaming. At least two parallel measurements of each 257 sample were analyzed.

258

259

2.10. Interfacial tension by surface tensiometer

260 The du Noüy ring method was used to measure the changes in the surface tension of

261 aqueous suspensions of functionalized nanoparticles as a function of concentration at room

262 temperature (du Noüy 1925). Distilled water and pure diesel oil were also measured as

263 references.

264 In the du Noüy method, a platinum wire ring lying in a plane parallel to the undisturbed

265 liquid surface is submerged in the liquid and then slowly withdrawn, while the net fluid

266 force on the ring is measured. The force $(F)$ that is required to raise the ring from the liquid

267 is measured and related to the liquid's surface tension $(\gamma)$. The maximum force $\left(F_{\max }\right)$ that

268 is obtained by the ring can be directly related to the interfacial tension using Equation 1:

269

$$
\boldsymbol{F}=2 \pi \cdot\left(\boldsymbol{r}_{i}+\boldsymbol{r}_{\boldsymbol{o}}\right) \cdot \boldsymbol{\gamma}
$$

270 where $r_{i}$ is the radius of the inner ring of the liquid film pulled, and $r_{0}$ is the radius of the 271 outer ring of the liquid film.

\section{3. Results and discussion}


275 Amphiphilic cellulose nanocrystals were produced from the fines fraction of the semi-

276 chemical pulp and Kraft pulp fibers (reference sample) by using combined oxidation and

277 reductive amination treatments. The semi-chemically produced corrugated board pulp was

278 first fractionated with a $150 \mu \mathrm{m}$ slot hole sieve to obtain a fraction containing only the fines

279 particles, and then analyzed to determine their average length and width, as well as size

280 distribution. The average (length-weighted) length and width of the hardwood pulp fibers,

281 determined using a Metso FiberLab image analyzer (Metso Automation, Helsinki, Finland),

282 were $0.65 \mathrm{~mm}$ and $21.2 \mu \mathrm{m}$, respectively. After screening the original sample, a different

283 fraction was obtained. The average length in the fines fraction was roughly half that of the

284 original pulp fibers, $0.30 \mathrm{~mm}$, while the average width was $16.2 \mu \mathrm{m}$. The fines fraction

285 consists of mainly split or cut fibers, or external fibrils, originating from the pulp refining

286 stage (Koskenhely 2008). The wood extractives, hemicellulose, and lignin contents of the

287 original semi-chemical pulp were $1.8 \%, 14.1 \%$, and $14.7 \%$, respectively, and, for the Kraft

288 pulp (reference sample), they were $0.08 \%, 24.7 \%$, and $0.4 \%$, respectively. Thus, the

289 cellulose content was $70.4 \%$ for the semi-chemical pulp and $74.8 \%$ for the Kraft pulp. The

290 wood extractives' content of the separated fines fraction was $0.1 \%$, content of

291 hemicelluloses was $4.8 \%$, and total lignin content was $37.3 \%$.

292 The chemical analysis revealed a high content of lignin in the fines fraction. The lignin 293 content of the original pulp was $14.7 \%$, but, in the fines fraction, the lignin content was $29437.3 \%$. A possible explanation of this phenomenon is that lignin is located in the outer layer 295 of the fibers and removed easily when fines are peeled from the fiber surface during the 296 pulp refining treatment. Due to the high lignin content, the fines fraction was expected to 
297 be naturally more hydrophobic than the Kraft pulp fibers, which increases its stabilization

298 potential in oil emulsification applications. Typically, chemical pulp contains less than $15 \%$

299 fines content (Ek et al. 2009) which causes problems in paper manufacture due to their

300 poor quality and binding ability. Therefore, they are normally rejected from the process.

\subsection{Chemical properties of bifunctionalized CNCS}

CNCs were liberated from the fines fraction of the semi-chemical pulp and Kraft pulp fibers

304 after combined oxidation and reductive amination treatments, followed by mechanical

305 homogenization. These chemical reaction routes were described in a previous publication

306 (Ojala et al. 2016). The aldehyde and carboxyl contents of the cellulose, after periodate and

307 chlorite oxidation, and the butylamino group contents of the nanocelluloses (CNC-LIG-1-2

308 and (NC-CHEM) are shown in Table 2. The initial aldehyde contents were similar for CNC-

309 LIG-1 and CNC-LIG-2 at $2.43 \mathrm{mmol} \mathrm{g}^{-1}$ and $2.62 \mathrm{mmol} \mathrm{g}^{-1}$, respectively, despite different 310 oxidation temperatures and chemical doses. It is possible that the high lignin content affects 311 negatively to the oxidation by consuming the oxidant for reactions of lignin (Gosselink et al.

312 2011). This assumption is supported by the fact that the CNC-CHEM (made from low-lignin

313 Kraft pulp) had a 32\% higher aldehyde content $\left(3.86 \mathrm{mmol} \mathrm{g}^{-1}\right)$ than CNC-LIG-2, despite a 314 similar oxidation treatment. On the other hand, the high aldehyde content of CNC-LIG-1 315 may indicate the presence of metals or other impurities that catalyze the reaction, and, 316 thus, result in a higher aldehyde content. The analyzed carboxyl contents revealed that the 317 conversion rates in the carboxylation reaction were $28.8 \%, 61.1 \%$, and $25.9 \%$ for CNC-LIG- 
318 1, CNC-LIG-2, and CNC-CHEM, respectively, while the $n$-butylamine contents of aminated

319 nanocelluloses were $0.85 \mathrm{mmol} \mathrm{g}^{-1}, 0.49 \mathrm{mmol} \mathrm{g}^{-1}$, and $0.86 \mathrm{mmol} \mathrm{g}^{-1}$, respectively. These

320 values correspond to the conversion rates of $35.0 \%, 18.7 \%$, and $22.3 \%$, respectively. The

321 residual aldehydes were converted into alcohol groups by the reductive agent used in the

322 amination reaction step.

323

324 Table 2. Aldehyde and the carboxyl contents of bifunctionalized CNC samples with $n$ -

325 butylamine groups attached.

326

327 3.3. Morphology of modified nanocelluloses

328 The morphology of the functionalized cellulose nanocrystals was visualized with TEM, and

329 image analysis was used to average the lengths and the widths of the individual CNCs from

330 the samples. The TEM images of isolated nanocrystals from semi-chemical pulp cellulose

331 are shown in Fig. 1. CNC-LIG-1 revealed rod-like, single crystalline CNCs, with lengths of 40

Sample Initial aldehydes Carboxylic acid content Amino group content

\begin{tabular}{cccc} 
& $\left(\mathbf{m m o l ~ g}^{-\mathbf{1}}\right)$ & $\left(\mathbf{m m o l ~ g}^{-\mathbf{1}}\right)$ & $(\mathbf{~ m m o l ~ g})$ \\
\hline CNC-LIG-1 & 2.43 & 0.7 & 0.85 \\
CNC-LIG-2 & 2.62 & 1.6 & 0.49 \\
CNC-CHEM & 3.86 & 1.0 & 0.86 \\
\hline
\end{tabular}

332 to $100 \mathrm{~nm}$ and lateral dimensions from 2 to $4 \mathrm{~nm}$ (Fig. 1). The images also revealed the

333 presence of nanofibrils in the samples, since all of the fibers did not disintegrate into 334 crystals, and the suspension was studied unpurified. The size distribution for the CNC-LIG-2 335 was wide, and some of the cellulose nanocrystals and nanofibers were agglomerated; still, 336 most of the material was liberated into crystalline form. The lengths varied from 
337

338

339

340

341

342

343

344

345

346

347

348

349

350

351

352

approximately 60 to $400 \mathrm{~nm}$, though it was not always obvious if it was one fibril or several crystals aligned. However, the average length of the CNC-LIG-2 was $160 \mathrm{~nm}$, and the lateral dimensions varied from 2 to $4 \mathrm{~nm}$. The CNC-CHEM made from Kraft cellulose was thoroughly liberated into individual crystals with similar size distributions, as reported earlier by Ojala et al. (2016), so the average length of one cellulose nanocrystal was approximately 60 to $70 \mathrm{~nm}$, and the lateral dimension was $3 \mathrm{~nm}$.
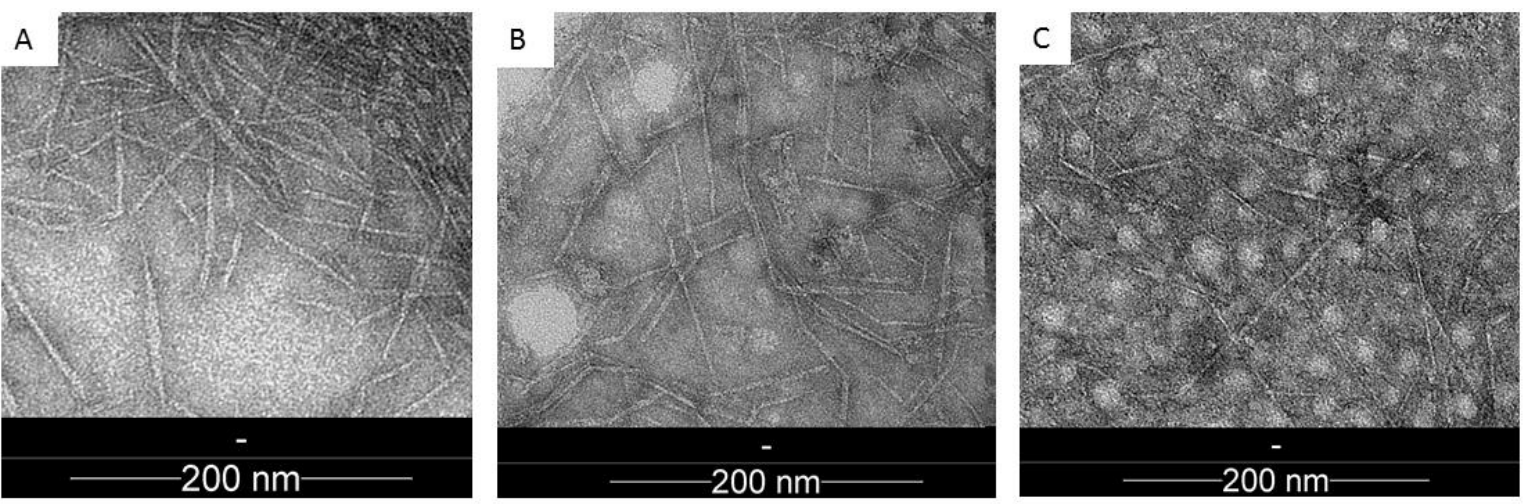

Figure 1. TEM images of a bifunctionalized CNC-CHEM (A) originated from Kraft pulp and CNC-LIG-1 and CNC-LIG-2 (B and C) made from a fines fraction of semi-chemical pulp.

\subsection{Emulsion creaming stability and droplet size measurements}

Diesel o/w emulsions were formed using cellulose nanocrystals as stabilizers. The o/w ratio remained at a constant $10 \%(\mathrm{w} / \mathrm{w})$ oil level during all experiments. The oil droplet sizes and their distribution from the emulsion were analyzed with the laser diffraction particle size analyzer. The distributions of oil droplet sizes are presented as volume versus size in Figure 
353 2. At a nanocellulose concentration of $0.05 \%$, the droplet sizes were in the range of $8.7-$

$354 \quad 13.6 \mu \mathrm{m}$ when measured at room temperature, while the reference emulsion, without any

355 added nanocellulose, had a droplet size of about $50 \mu \mathrm{m}$ and was unstable. The measured

356 average droplet sizes and standard deviations are shown in Table 3. The CNC-LIG-2 in a

$357 \quad 0.05 \%$ concentration resulted in the smallest oil droplet size and performed well in cold

358 conditions. A decrease in the dispersing temperature had an obvious effect on the particle

359 sizes of CNC-CHEM; the average particle size increased from 13.6 to $22.2 \mu \mathrm{m}$. However, the

360 higher nanocellulose concentration of $0.1 \%$ compensated for the particle size increase

361 caused by the lower temperature, and the droplet sizes were the same as those of the

$3620.05 \%$ concentration at room temperature. CNC-LIG-1 and CNC-LIG-2 did not show any

363 change in the stabilized oil droplet size at the lower temperature according to mean droplet

364 size. However, the distribution of the oil droplet sizes in the Figure 2 shows the higher

365 amount of smaller droplets when CNC-LIG-1 and CNC-LIG-2 are used.

\begin{tabular}{ccccc}
\hline $\begin{array}{c}\text { CNC used in } \\
\text { emulsion }\end{array}$ & $\begin{array}{c}\text { Concentration } \\
(\%)\end{array}$ & $\begin{array}{c}\text { Temperature } \\
\left({ }^{\circ} \mathbf{C}\right)\end{array}$ & $\begin{array}{c}\text { Mean } \\
(\mu \mathrm{m})\end{array}$ & $\begin{array}{c}\text { Standard deviation } \\
(\mu \mathrm{m})\end{array}$ \\
\hline CNC-LIG-1 & 0.05 & +20 & 11.0 & 7.7 \\
& 0.05 & +5 & 13.1 & 9.2 \\
& 0.10 & +5 & 8.3 & 6.4 \\
\hline CNC-LIG-2 & 0.05 & +20 & 8.7 & 6.0 \\
& 0.05 & +5 & 7.2 & 5.7 \\
\hline CNC-CHEM & 0.10 & +5 & 7.2 & 5.6 \\
& 0.05 & +20 & 13.6 & 7.1 \\
& 0.05 & +5 & 22.2 & 13.3 \\
& 0.10 & +5 & 12.4 & 8.7 \\
\hline
\end{tabular}



Table 3. Average oil droplet sizes of o/w emulsions in different CNC -concentrations and temperatures.

370 Visual analysis of the sizes of the oil droplets was done through stereomicroscopy from the

371 readily made o/w emulsions. A small aliquot of the emulsion was placed on a glass slide and 372 covered with a glass coverslip. The images (Fig.3) of the oil droplets were observed, and size 373 results were comparable to those obtained from the particle size analysis.
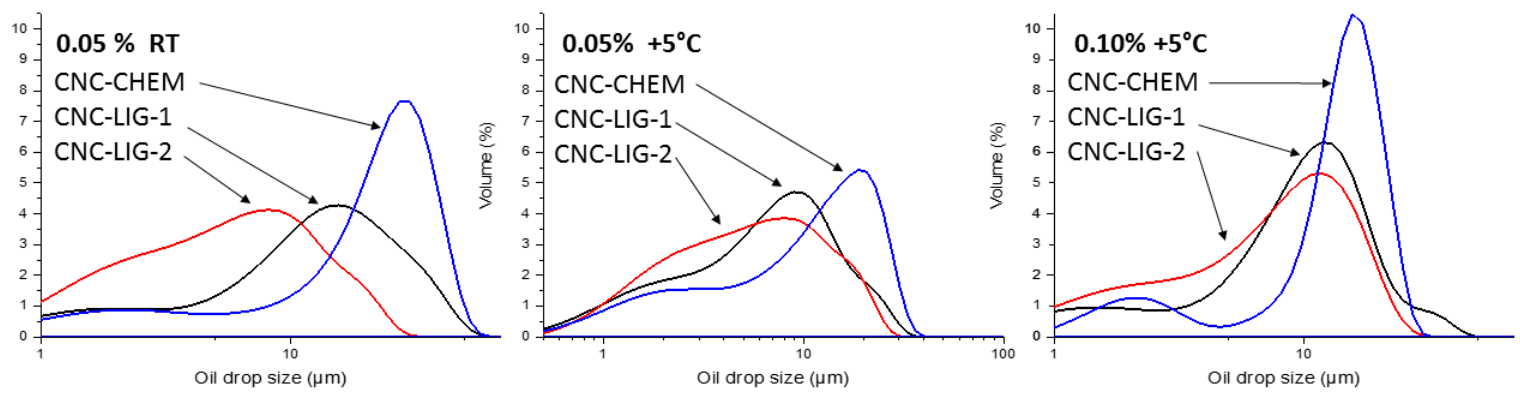

375 Figure 2. Droplet diameter distributions of emulsions stabilized by CNC-LIG-1, CNC-LIG-2 376 and CNC-CHEM in concentrations of $0.05 \%$ and $0.10 \%$ (on the left). Distributions were 377 analyzed at both room temperature and at $+5^{\circ} \mathrm{C}$.
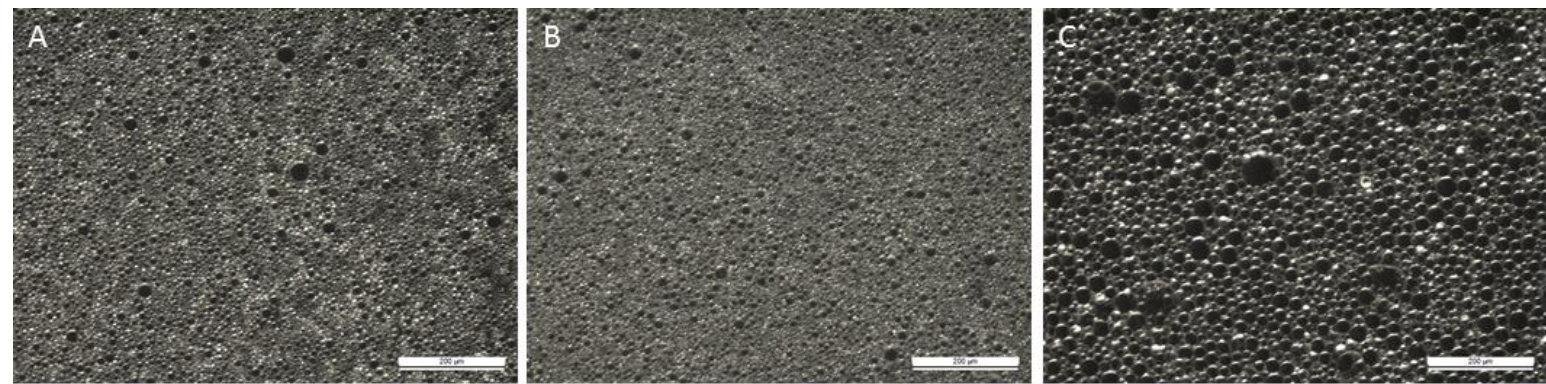

Figure 3. Stereomicroscopy images of CNC-stabilized o/w emulsion at $0.05 \%$ (w/w) CNC-LIG-

1 (A), CNC-LIG-2 (B) and CNC-CHEM (C) using 10\% (w/w) of oil content. 
381 O/w emulsion creaming stability was determined with the LUMifuge analytical centrifuge

382 by monitoring the formation of the creaming layer. The stability analysis was done on a

$3830.05 \%(w / w)$ nanoparticle concentration at room temperature and in two different CNC 384 concentrations, $0.05 \%$ and $0.1 \%(\mathrm{w} / \mathrm{w})$, at a colder $+5{ }^{\circ} \mathrm{C}$ temperature. Figure 4 illustrates 385 the stability behavior against creaming of emulsions as a function of time. The stability at 386 room temperature with a $0.05 \%(w / w)$ CNC concentration was clearly improved since the 387 CNC-LIG-1 reached the final 43\% transmission rate and the CNC-LIG-2 slowly increased only 388 at $30 \%$ transmittance during the analysis time. The creaming occurred with CNC-CHEM soon 389 after the analysis started (at a concentration of $0.05 \%$ ), the transmission was approximately $39050 \%$ after 10 min of centrifugation, and this sample finally reached a $70 \%$ transmission rate.

391 The CNCs obtained from the fines fraction of the semi-chemical pulp seemed to work better 392 compared to the Kraft cellulose nanocrystals, probably due to more naturally hydrophobic 393 surface characteristics. For those CNCs, the creaming was slower (CNC-LIG-1 and CNC-LIG3942 2), and no droplet coalescence occurred.

396 Usually, creaming increases the risk of oil droplet coalescence; this means that the forces, 397 which keep the surface between the oil droplets thick enough, weaken and the oil droplets 398 fuse together forming larger droplets (Tadros 2013). In this work, the stability of all studied 399 samples improved in the colder conditions. The lower temperature decreased the thermal 400 motion of droplets and increased the viscosity of fluids, and it therefore reduced the rate 401 of phase separation. The creaming occurred, but the separation was very slow, especially 
402 with CNC-LIG-2. The final transmission rate for CNC-LIG-2, during analysis time, was $5 \%$.

403 CNC-LIG-1 did not perform as well at a concentration of $0.05 \%(w / w)$, but increasing the 404 concentration of CNCs to $0.1 \%(\mathrm{w} / \mathrm{w})$ resulted in improved stability and approximately $10 \%$ 405 final transmission. The CNC-CHEM had the most significant differences between different 406 temperatures and concentrations of CNCs, but it still performed as well as the CNC-LIG-1 407 and CNC-LIG-2 when the dispersant concentration was $0.1 \%$.

408 In the figure 4, the differences in emulsion stability between different CNC -samples in 409 same physical conditions are clearly shown. It can be detected that in lower concentration 410 (0.05\% CNC), CNC-LIG-1 and CNC-LIG-2 performed better than CNC-CHEM, as the 411 transmission level was lower ie. the creaming occurred more slowly. In $0.10 \%(w / w) ~ C N C$ 412 concentration there were no differences in transmittance.
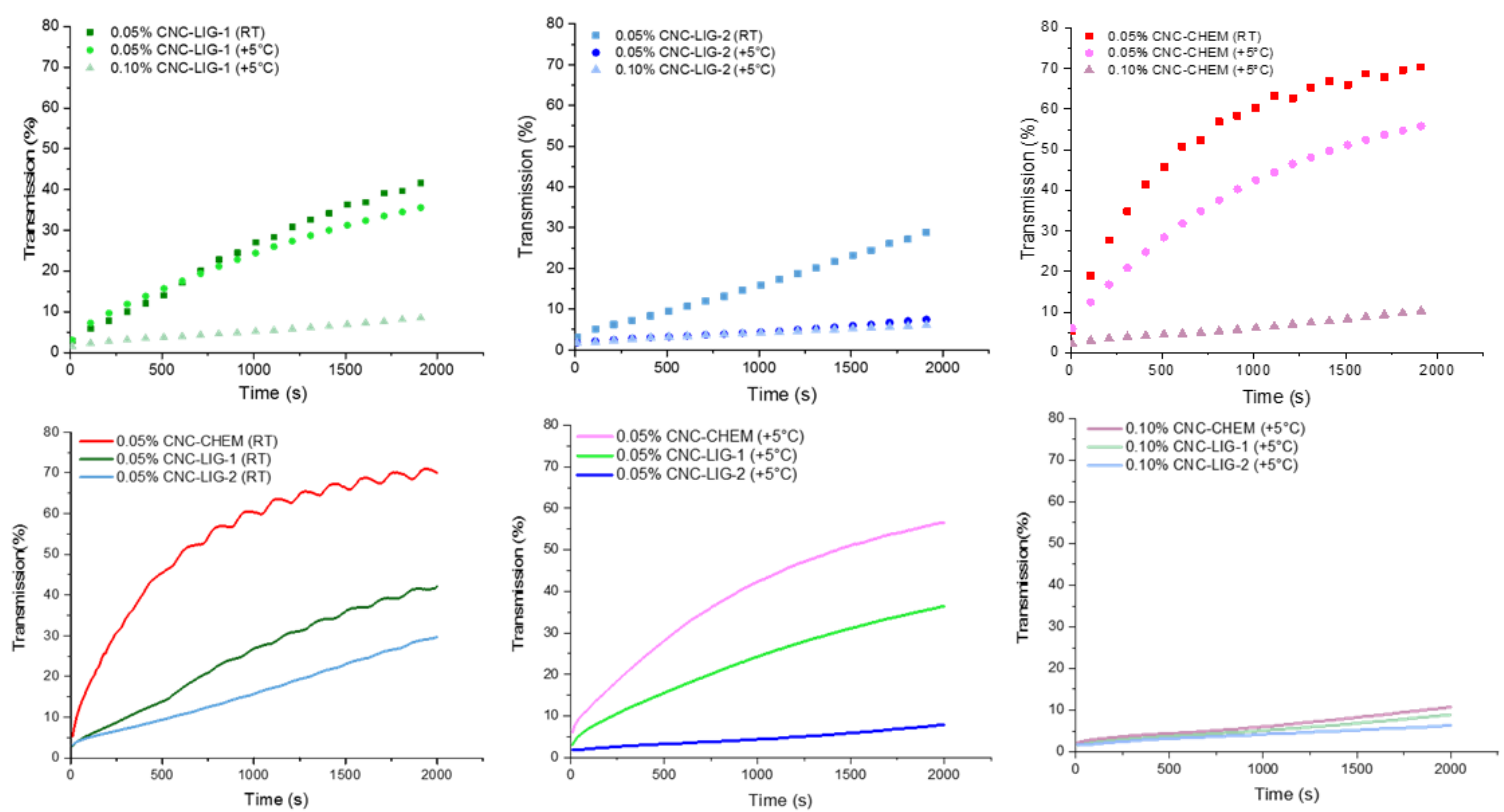
414 Figure 4. Transmission against time for o/w emulsions stabilized with butylamino-

415 functionalized nanocellulose dispersants during centrifugation at 1,200 rpm rotational

416 speed for 30 minutes. CNC-LIG-1 (up-left), CNC-LIG-2 (up-middle), and CNC-CHEM (up-right)

417 were studied in $0.05 \%(\mathrm{w} / \mathrm{w})$ and $0.10 \%(\mathrm{w} / \mathrm{w})$ concentrations at both room temperature $418\left(+20^{\circ} \mathrm{C}\right)$ and $+5^{\circ} \mathrm{C}$.

\subsection{Surface tension reduction using dispersants}

421 A variety of dispersants are used in applications where interfacial tension between two

422 phases is too high to form stable emulsions. Hydrophobized cellulose CNC suspension can

423 act as a dispersant and change a liquid's wetting characteristics by altering the surface

424 tension at liquid interfaces. In this work, the surface tensions, in different concentrations

425 for the series of samples with butylamino-functionalized CNCs in water, were determined

426 with the du Noüy ring method.

427 Figure 5 shows the decreasing surface tension $\gamma_{A W}$ of both bifunctionalized CNCs when

428 added to the water already at low concentrations. The initial value for pure water was 72.4

$429 \mathrm{mN} / \mathrm{m}$ at room temperature. The dispersant concentrations varied from $0.01-0.1 \% \mathrm{w} / \mathrm{v}$,

430 and, compared to plain water, the surface tension decreased $11 \%$ when CNC-LIG-1 was

431 mixed with water at a $0.1 \%(\mathrm{w} / \mathrm{v})$ concentration and $26 \%$ when the CNC-LIG-2 concentration

432 was at $0.1 \%(\mathrm{w} / \mathrm{v})$ in water. This reduction of interfacial tension improves the dispersion of

433 oil, and smaller oil droplet sizes in the emulsion are formed. 


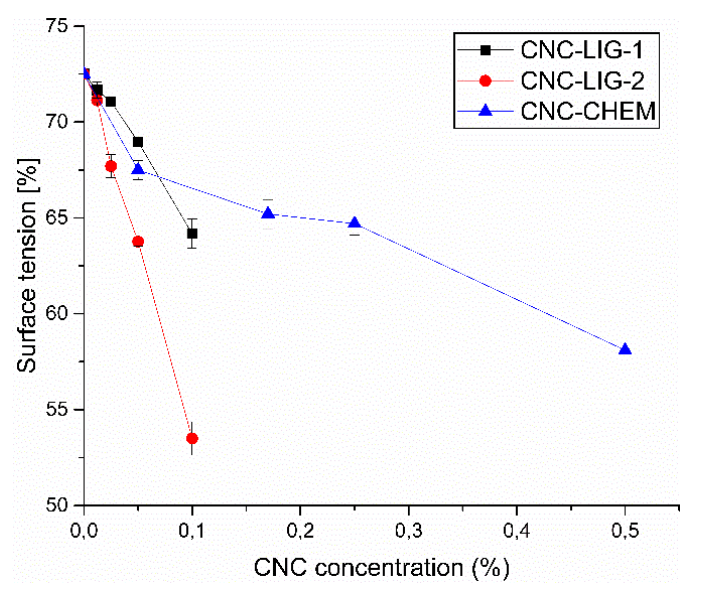

435 Figure 5. Air/deionized water surface tension at different CNC concentrations. In 436 comparison, pure deionized water $\gamma_{\text {AW }}$ was $72.4 \mathrm{mN} / \mathrm{m}$ at $24{ }^{\circ} \mathrm{C}$, while surface tension with $437 \quad 0.10 \%(\mathrm{w} / \mathrm{w})$ CNC-LIG-1 was $64.2 \mathrm{mN} / \mathrm{m}$ and with $0.10 \%(\mathrm{w} / \mathrm{w})$ CNC-LIG-2 $53.5 \mathrm{mN} / \mathrm{m}$.

To study the effect on the marine environment of the cellulose-based biochemicals and

441 their effectiveness on o/w emulsion stability, synthetic seawater was prepared, and oil

442 droplet size and emulsion stability were measured from emulsions stabilized with all CNC

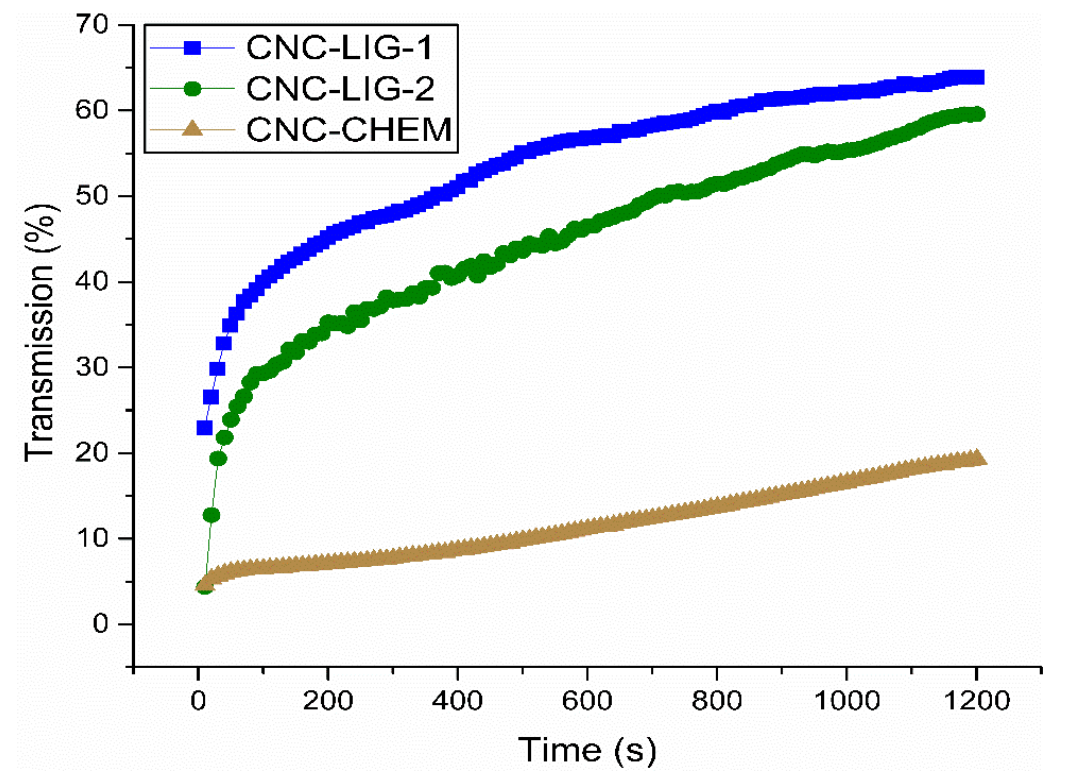


443

444

445

446

447

448

449

450

451

452

453

454

455

456

457

458

459

460

461 salt effects only on creaming that is not a negative phenomenon when application in

462 enhanced oil recovery is considered.

samples at a concentration of $0.05 \%$. Immediately after emulsification, all samples seemed to be uniform, and emulsion formation was good. However, the creaming effect was detected very soon after emulsification, which was in agreement with the stability analysis performed with the LUMifuge. No visual droplet coalescence was observed in any of the studied samples. In this experiment, the results showed that the CNC-CHEM performed best in saline conditions. The phase separation was slow, and the emulsion stability remained good. The final transmission rate for CNC-CHEM was approximately $20 \%$. These results are presented in Figure 6.

Figure 6. Emulsion stability in cold, synthetic $\left(+5^{\circ} \mathrm{C}\right)$ seawater at a low concentration of CNCs at $0.05 \% \mathrm{w} / \mathrm{w}$.

The results revealed that the effect of low temperature was not as significant as the saline environment on the oil droplet size or on the emulsion stability. The CNC-LIG-2 made from the fines fraction performed well in the $0.05 \%$ concentration when standard cold water was used, but the $3.5 \%$ salinity negatively affected the stability of the emulsion. On the other hand, CNC-CHEM performed better in the synthetic seawater when already at a low concentration (0.05\%). It is probably due to weaker interactions with oil and lignin-rich CNCs in this $\mathrm{pH}$ and high salinity. Since no coalescence of oil was visually observed, it is assumed, that the steric barrier is good enough to prevent oil drops separated and the background

\section{Conclusions}


464 In this work, unpurified, lignin-rich reject cellulose fiber fines were used to produce a novel 465 nanoparticle dispersant containing modified CNCs. A chemical modification route was used

466 to fabricate bifunctionalized nanocelluloses to improve diesel oil dispersing and 467 simultaneous oil droplet stabilization in an o/w emulsion. This study indicated that there is 468 the potential to also utilize reject cellulose material, i.e., fiber fines, in the fabrication of bio469 based dispersing and stabilizing agents for o/w emulsion stabilization. Having demonstrated 470 the utilization of cellulose-based nanoparticles as a raw material in the fabrication of a bio471 based dispersant, our study indicates that future studies should further investigate the 472 interactions between cellulose nanoparticles and dispersed oil droplets. Moreover, the 473 stabilization mechanisms in deionized water and in saline environments are interesting, 474 since the low temperature was not as significant as the saline environment in affecting the 475 oil droplet size or the stability characteristics of the emulsion in standard water. The high 476 lignin content in pulp promotes the natural hydrophobicity, and, therefore, the wood 477 possesses properties that suggest the potential for other applications such as the oil spill 478 response. Although some interactions between oil droplets and amphiphilic nanoparticles 479 can be explained by steric stabilization, the effect of electrostatic repulsions on emulsion 480 stability still requires more detailed study.

\section{Acknowledgements}


484

485

486

487

488

489

490

491

492

493

494

495

496

497

498

499

500

501

502

503

504

505

506

507

508

509

510

511

512

This study was funded by the Academy of Finland (283187). The support of the Ahti Pekkala Foundation and the Tiina and Antti Herlin Foundation is gratefully acknowledged. The contribution of Mr. Mikael Karjalainen in the raw material characterization and the experience of Dr. Ilkka Miinalainen in TEM measurements are also much appreciated. We thank Neste Oyj Finland for providing the marine diesel oil sample for the experiments.

\section{References}

Al-Majed AA, Adebayo AR, Hossain ME (2012) A sustainable approach to controlling oil spills. J Environ Manage 113:213-227. doi: 10.1016/j.jenvman.2012.07.034

Chevalier Y, Bolzinger M-A (2013) Emulsions stabilized with solid nanoparticles: Pickering emulsions. Colloids Surf Physicochem Eng Asp 439:23-34. doi:

10.1016/j.colsurfa.2013.02.054

Daza EA, Misra SK, Scott J, et al (2017) Multi-Shell Nano-CarboScavengers for Petroleum Spill Remediation. Sci Rep 7:41880. doi: 10.1038/srep41880

Du Nouy PL (1925) AN INTERFACIAL TENSIOMETER FOR UNIVERSAL USE. J Gen Physiol 7:625-631. doi: 10.1085/jgp.7.5.625

Ek M, Gellerstedt G, Henriksson G (2009) Pulping Chemistry and Technology. In: Pulp and Paper Chemistry and Technology. De Gruyter, Berlin,

García A, Gandini A, Labidi J, et al (2016) Industrial and crop wastes: A new source for nanocellulose biorefinery. Ind Crops Prod 93:26-38. doi: 10.1016/j.indcrop.2016.06.004

George-Ares A, Clark JR (2000) Aquatic toxicity of two Corexit ${ }^{\circledR}$ dispersants. Chemosphere 40:897906. doi: 10.1016/S0045-6535(99)00498-1

Gharehkhani S, Sadeghinezhad E, Kazi SN, et al (2015) Basic effects of pulp refining on fiber properties-A review. Carbohydr Polym 115:785-803. doi: 10.1016/j.carbpol.2014.08.047

Gosselink RJA, van Dam JEG, de Jong E, et al (2011) Effect of periodate on lignin for wood adhesive application. Holzforschung. doi: 10.1515/hf.2011.025

Guodong Q, Yupeng Z, Xuhe R, Jie C (2015) Research on Development and Effectiveness Evaluation Technology of New Environment-friendly Oil Spill Dispersant. Aquat Procedia 3:245-253. doi: 10.1016/j.aqpro.2015.02.218 
513

514

515

516

517

518

519

520

521

522

523

524

525

526

527

528

529

530

531

532

533

534

535

536

537

538

539

540

541

542

543

544

545

546

547

Hu Z, Ballinger S, Pelton R, Cranston ED (2015a) Surfactant-enhanced cellulose nanocrystal Pickering emulsions. J Colloid Interface Sci 439:139-148. doi: 10.1016/j.jcis.2014.10.034

Hu Z, Patten T, Pelton R, Cranston ED (2015b) Synergistic Stabilization of Emulsions and Emulsion Gels with Water-Soluble Polymers and Cellulose Nanocrystals. ACS Sustain Chem Eng 3:1023-1031. doi: 10.1021/acssuschemeng.5b00194

Jiang Y, Liu X, Chen Y, et al (2014) Pickering emulsion stabilized by lipase-containing periodic mesoporous organosilica particles: A robust biocatalyst system for biodiesel production. Bioresour Technol 153:278-283. doi: 10.1016/j.biortech.2013.12.001

Kalashnikova I, Bizot H, Bertoncini P, et al (2013) Cellulosic nanorods of various aspect ratios for oil in water Pickering emulsions. Soft Matter 9:952-959. doi: 10.1039/C2SM26472B

Kalashnikova I, Bizot H, Cathala B, Capron I (2011) New Pickering emulsions stabilized by bacterial cellulose nanocrystals. Langmuir ACS J Surf Colloids 27:7471-7479. doi: 10.1021/la200971f

Kari Koskenhely (2008) Refining of chemical pulp fibers. In: Papermaking Part 1, Stock preparation and wet end. Finnish paper engineers' association,

Katz S, Beatson RP, Scallan AM (1984) The determination of strong and weak acidic groups in sulfite pulps. Sven. Papperstidning 795-816.

Kester DR, Duedall IW, Connors DN, Pytkowicz RM (1967) Preparation of artificial seawater. Limnol Oceanogr 12:176-179. doi: 10.4319/lo.1967.12.1.0176

Nyankson E, DeCuir MJ, Gupta RB (2015) Soybean Lecithin as a Dispersant for Crude Oil Spills. ACS Sustain Chem Eng 3:920-931. doi: 10.1021/acssuschemeng.5b00027

Ojala J, Sirviö JA, Liimatainen H (2016) Nanoparticle emulsifiers based on bifunctionalized cellulose nanocrystals as marine diesel oil-water emulsion stabilizers. Chem Eng J 288:312-320. doi: 10.1016/j.cej.2015.10.113

Orelma H, Tanaka A, Rautkoski H, et al (2017) Mechanically ground softwood fines as a raw material for cellulosic applications. Cellulose 24:3869-3882. doi: 10.1007/s10570-0171403-x

Pi G, Li Y, Bao M, et al (2016) Novel and Environmentally Friendly Oil Spill Dispersant Based on the Synergy of Biopolymer Xanthan Gum and Silica Nanoparticles. ACS Sustain Chem Eng 4:3095-3102. doi: 10.1021/acssuschemeng.6b00063

Prince RC (2015) Oil Spill Dispersants: Boon or Bane? Environ Sci Technol 49:6376-6384. doi: 10.1021/acs.est.5b00961

Rattaz A, Mishra SP, Chabot B, Daneault C (2011) Cellulose nanofibres by sonocatalysed-TEMPOoxidation. Cellulose 18:585-593. doi: 10.1007/s10570-011-9529-8

Saha A, Nikova A, Venkataraman P, et al (2013) Oil Emulsification Using Surface-Tunable Carbon Black Particles. ACS Appl Mater Interfaces 5:3094-3100. doi: 10.1021/am3032844 
548

549

550

551

552

553

554

555

556

557

558

559

560

561

562

563

564

565

566

567

568
Silva MC, Lopes OR, Colodette JL, et al (2008) Characterization of three non-product materials from a bleached eucalyptus kraft pulp mill, in view of valorising them as a source of cellulose fibres. Ind Crops Prod 27:288-295. doi: 10.1016/j.indcrop.2007.11.005

Sirvio J, Hyvakko U, Liimatainen $\mathrm{H}$, et al (2011) Periodate oxidation of cellulose at elevated temperatures using metal salts as cellulose activators. Carbohydr Polym 83:1293-1297. doi: 10.1016/j.carbpol.2010.09.036

Tadros TF (ed) (2013) Emulsion formation and stability. Wiley-VCH, Weinheim

Wang W, Zheng Y, Lee K (2013) Chemical dispersion of oil with mineral fines in a low temperature environment. Mar Pollut Bull 72:205-212. doi: 10.1016/j.marpolbul.2013.03.042

Xhanari K, Syverud K, Stenius P (2011) Emulsions Stabilized by Microfibrillated Cellulose: The Effect of Hydrophobization, Concentration and O/W Ratio. J Dispers Sci Technol 32:447-452. doi: 10.1080/01932691003658942

Yu G, Dong J, Foster LM, et al (2014) Breakup of Oil Jets into Droplets in Seawater with Environmentally Benign Nanoparticle and Surfactant Dispersants. Ind Eng Chem Res 141223085223001. doi: 10.1021/ie503658h

Zeinstra-Helfrich M, Koops W, Murk AJ (2015) The NET effect of dispersants - a critical review of testing and modelling of surface oil dispersion. Mar Pollut Bull 100:102-111. doi: 10.1016/j.marpolbul.2015.09.022

Zhang Y, Chen D, Ennis AC, et al (2013) Chemical dispersant potentiates crude oil impacts on growth, reproduction, and gene expression in Caenorhabditis elegans. Arch Toxicol 87:371-382. doi: 10.1007/s00204-012-0936-x 